\title{
Pre-onset auroral signatures and subsequent development of substorm auroras: a development of ionospheric loop currents at the onset latitudes
}

\author{
O. Saka ${ }^{1}$, K. Hayashi ${ }^{2}$, and M. Thomsen ${ }^{3}$ \\ ${ }^{1}$ Office Geophysik, Ogoori, Japan \\ ${ }^{2}$ University of Tokyo, Tokyo, Japan \\ ${ }^{3}$ Los Alamos National Laboratory, Los Alamos, NM, USA \\ Correspondence to: O. Saka (saka.o@nifty.com)
}

Received: 26 February 2014 - Revised: 14 July 2014 - Accepted: 14 July 2014 - Published: 22 August 2014

\begin{abstract}
Substorm auroras observed on 17 January 1994 were localized within the field of view of an all-sky imager installed at Dawson City (DWS, $65.7^{\circ}$ ILAT). In association with the enhancement of the anti-sunward convection in the polar cap and the ion flux enhancement in $1-6 \mathrm{keV}$ at geosynchronous altitudes, a wave-like structure propagating equatorward to the onset latitudes with a high wave number in azimuth ( $m \sim 76, T \sim 120 \mathrm{~s}$ ) was observed $30 \mathrm{~min}$ prior to the activation in the equatorward latitudes. The activation of the auroras in the equatorward latitudes and the subsequent poleward expansion lasted for approximately 6 min until a diffuse aurora formed. The auroras in the last 6 min were isolated and localized within the field of view of DWS, from $400 \mathrm{~km}$ west to $400 \mathrm{~km}$ east, and accompanied the magnetic pulse at the optical station. The magnetic pulse is interpreted by the propagating ionospheric current loop with a size comparable to the isolated auroras $(\sim 1000 \mathrm{~km})$. We conclude that the wave-like structures in the pre-onset interval relate to the intrusion of the plasma-sheet plasmas from the tail by the convection. The plasmas from the tail eventually developed the ionospheric loop currents at the onset latitudes, in association with the triggering of the bead-like rippling of auroras and subsequent breaking out from the onset latitudes.
\end{abstract}

Keywords. Ionosphere (auroral ionosphere) - magnetospheric physics (auroral phenomena; storms and substorms)

\section{Introduction}

Pi2 pulsations are periodic (50-170s) oscillations of short duration $(\sim 15 \mathrm{~min})$ observed in geomagnetic fields, particle flux, and in auroral luminosity related to the substorm expansion (Saka et al., 1999; Solovyev et al., 2000; Shiokawa et al., 2002). Periodic intensifications or propagations of field-aligned currents in a substorm current wedge (SCW) were proposed as the source of oscillations of the geomagnetic fields (Sakurai and McPherron, 1983; Baumjohann and Glassmeier, 1984; Lester et al., 1983; Rostoker and Samson, 1981; Samson, 1982). The periodic intensification of the electrojet, an ionospheric part of the SCW, is considered a source of the Pi2 signals associated with the auroral intensifications in the expansion phase (Nishimura et al., 2012). It is argued, however, that the SCW is composed of a number of localized current elements. Averaged current elements may be described as the traditional view of the SCW (Rae et al., 2013).

Meanwhile, one of the features of a high-latitude Pi2 is its longitudinal propagation with a finite wave number of $m$ (Samson and Harrold, 1985; Uozumi et al., 2004; Saka et al., 2012a). Here $m$ denotes azimuthal wave numbers encircling the Earth. For a finite $m$, the poloidal (meridional) and the toroidal (azimuthal) components of the field line oscillations couple in the magnetosphere. In particular, the poloidal mode becomes guided along the field lines for short-lived, transient oscillations, where the oscillations may not last long enough to establish true cavity modes (Radoski, 1967, 1974). All disturbances tend to become guided along the field lines because parallel component diminishes with time, which is referred to 
as a cavity/wave-guide mode (Allan et al., 1996). Compressional inputs with azimuthal wave number $m$ imposed at the outer boundary generate a cavity/wave-guide mode in the inner magnetosphere (Allan et al., 1985; Lee and Lysak, 1991; Lee, 1998). The cavity/wave-guide mode is used to explain Pi2s, both within the plasmasphere (Takahashi et al., 1992 and references in Keiling and Takahashi, 2011) and outside the plasmapause (Kim et al., 2005; Teramoto et al., 2008).

These two Pi2 models, an ionospheric current model and a cavity/wave-guide model, have been discussed individually. To reconcile those two models, an excitation of the current vortex in the ionosphere by a guided poloidal wave was proposed (Saka et al., 2012b). The current vortex in the ionosphere can also be regarded as current flows established by the currents carried by the poloidally polarized waves (Southwood and Hughes, 1985). The poloidal waves can be excited in the magnetospheric equatorial plane by the convection surge (Quinn and Southwood, 1982) or by the diamagnetic currents flowing eastward associated with the substorm ion injection (Saka et al., 2012b). The individual current vortex may form the current element and constitute a large-scale current system when the multiple vortices congregate (Amm et al., 2002). The ionospheric flow vortex associated with the $\mathrm{Pi} 2$ pulsations was reported using radars in the auroral zone (Sutcliffe and Nielsen, 1990, 1992; Bradshaw and Lester, 1997).

In this report, substorm auroras were studied beginning from the pre-onset interval to the subsequent development of auroras. This case study shows that fresh plasmas from the tail eventually developed the vortical currents in the ionosphere.

\section{Observation}

\subsection{Aurora and ground magnetometer observations}

In this analysis, we used the fluxgate magnetometer data from high-latitude ground stations of ERK, BRW, KOT, CMO, DWS, SIT, YKC, FSI, and FMC as well as lowlatitude stations of GUA and PFO, and the plasma measurements by the Magnetospheric Plasma Analyzer (MPA) on board the L9 satellite. Refer to Birn et al. (1997) for details regarding MPA instruments. The invariant latitudes and the MLT (at 10:00 UT) of the ground stations and the satellite footprints calculated by the IGRF (International Geomagnetic Reference Field) model are listed in Table 1.

Figure 1 depicts, from top to bottom, a keogram crossing 150 km west of DWS for the interval 09:00-11:00 UT, 17 January 1994 and the fluxgate magnetometer data from 09:00 to 11:00 UT for stations in the auroral zone (BRW, KOT, CMO, DWS, SIT, FSI, YKC, and FMC) and in the polar cap (ERK). $H$ component (pointing magnetic north) is plotted for the stations in the auroral zone, and the dawnto-dusk component (positive duskward) for the polar cap
Table 1. Invariant latitudes and MLT of ground stations and satellite footprints at 10:00 UT.

\begin{tabular}{lrr}
\hline Station name & $\begin{array}{r}\text { Inv. Lat. } \\
\text { (deg.) }\end{array}$ & $\begin{array}{r}\text { MLT } \\
(\text { 10:00 UT) }\end{array}$ \\
\hline ERK (Eureka) & 88.7 & 3.0 \\
BRW (Barrow) & 69.7 & 22.0 \\
KOT (Kotzebue) & 64.1 & 22.0 \\
CMO (College) & 64.7 & 22.9 \\
L9 (LANL 1989-046) & 66.9 & 23.1 \\
DWS (Dawson City) & 65.7 & 23.4 \\
SIT (Sitka) & 59.5 & 24.0 \\
FSI (Fort Simpson) & 67.4 & 0.8 \\
YKC (Yellow Knife) & 69.0 & 1.4 \\
FMC (Fort McMurray) & 64.4 & 1.8 \\
GUA (Guam) & 4.5 & 19.7 \\
PFO (PFO) & 39.5 & 1.7 \\
\hline
\end{tabular}

station. The vertical scale in the keogram shows the northsouth distance in kilometers from the DWS latitudes. An allsky image taken by the panchromatic CCD imager was used. The negative bays were observed at YKC, CMO, DWS, and FSI, commencing at 09:45, 09:48, 09:48, and 09:47 UT, respectively. The onset of the negative bay at DWS is correlated with the occurrence of an auroral breakup at 09:50 UT, as displayed in the top panel. At that time, YKC was located far east of the field of view of the all-sky imager. FSI was at the eastern edge of the field of view. CMO was in the field of view. Consecutive auroral breakups occurred at 10:35 and 10:42 UT. The peak of the negative bay occurred at the second breakup (10:42 UT). However, the auroras appeared vague because of clouds over the optical station.

We focused on the negative bay and the auroral breakup associated with the first dotted line (09:53 UT). The longitudinal extent of this breakup event is shown in Fig. 2 by longitudinal scanning from $900 \mathrm{~km}$ west to $900 \mathrm{~km}$ east of DWS along $128 \mathrm{~km}$ south of DWS $(128 \mathrm{~km} \mathrm{~S})$, above DWS $(0 \mathrm{~km} \mathrm{~N})$, and $100 \mathrm{~km}$ north of DWS $(100 \mathrm{~km} \mathrm{~N})$. The auroral breakup and following poleward expansion occurred in the sector between $400 \mathrm{~km}$ west $(\mathrm{W} 400 \mathrm{~km})$ and $400 \mathrm{~km}$ east $(\mathrm{E} 400 \mathrm{~km})$ from DWS for $128 \mathrm{~km} \mathrm{~S}$, in the sector W400 km and $E 400 \mathrm{~km}$ for $0 \mathrm{~km} \mathrm{~N}$, and in the sector $\mathrm{W} 400 \mathrm{~km}$ and $\mathrm{E} 200 \mathrm{~km}$ for $100 \mathrm{~km} \mathrm{~N}$. The pulsating auroras were activated to the west and east of the breakup sector. We conclude that the auroral breakup of the 17 January 1994 event occurred within the longitudes between $400 \mathrm{~km}$ east to $400 \mathrm{~km}$ west from DWS. The localization of the breakup sector can also be observed in the magnetogram in Fig. 1, where the amplitudes of the negative bay decreased by an order of magnitude at the adjoining stations of FSI and KOT, located $800 \mathrm{~km}$ to the east and $950 \mathrm{~km}$ west, respectively. The amplitudes decreased by half at CMO, located $410 \mathrm{~km}$ west of DWS. At BRW (69.7 $\left.7^{\circ} \mathrm{ILAT}\right)$ and at SIT $\left(59.5^{\circ} \mathrm{ILAT}\right)$, there was no clear sign of the negative bay. 

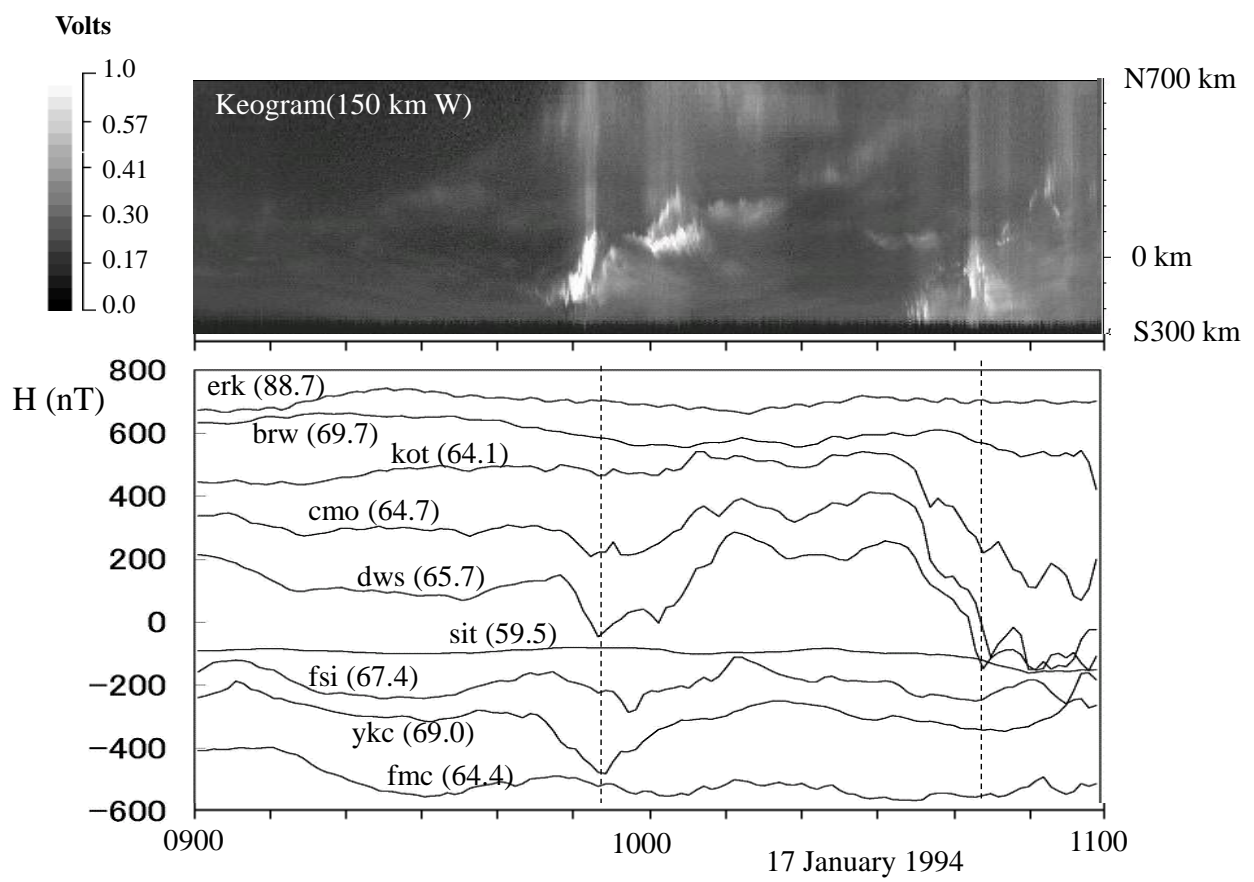

Figure 1. From top to bottom: 2-hour plots from 09:00 to 11:00 UT for 17 January 1994, showing a keogram along geomagnetic meridians located $150 \mathrm{~km}$ west of DWS (Keogram $(150 \mathrm{~km} \mathrm{~W})$ ) and the $H$ component of the magnetic field in $\mathrm{nT}$ from eight stations in the auroral zone (BRW, KOT, CMO, DWS, SIT, FSI, YKC, FMC) and dawn-to-dusk component from the polar cap station (ERK). The vertical dotted lines indicate peaks of the negative bay of DWS at 09:53 and 10:42 UT. The time resolution of the magnetogram is 1 min. The aurora gray level is plotted to the left in volts, where three pieces of polygonal line conversion was used to make suppressions for high and low voltages.

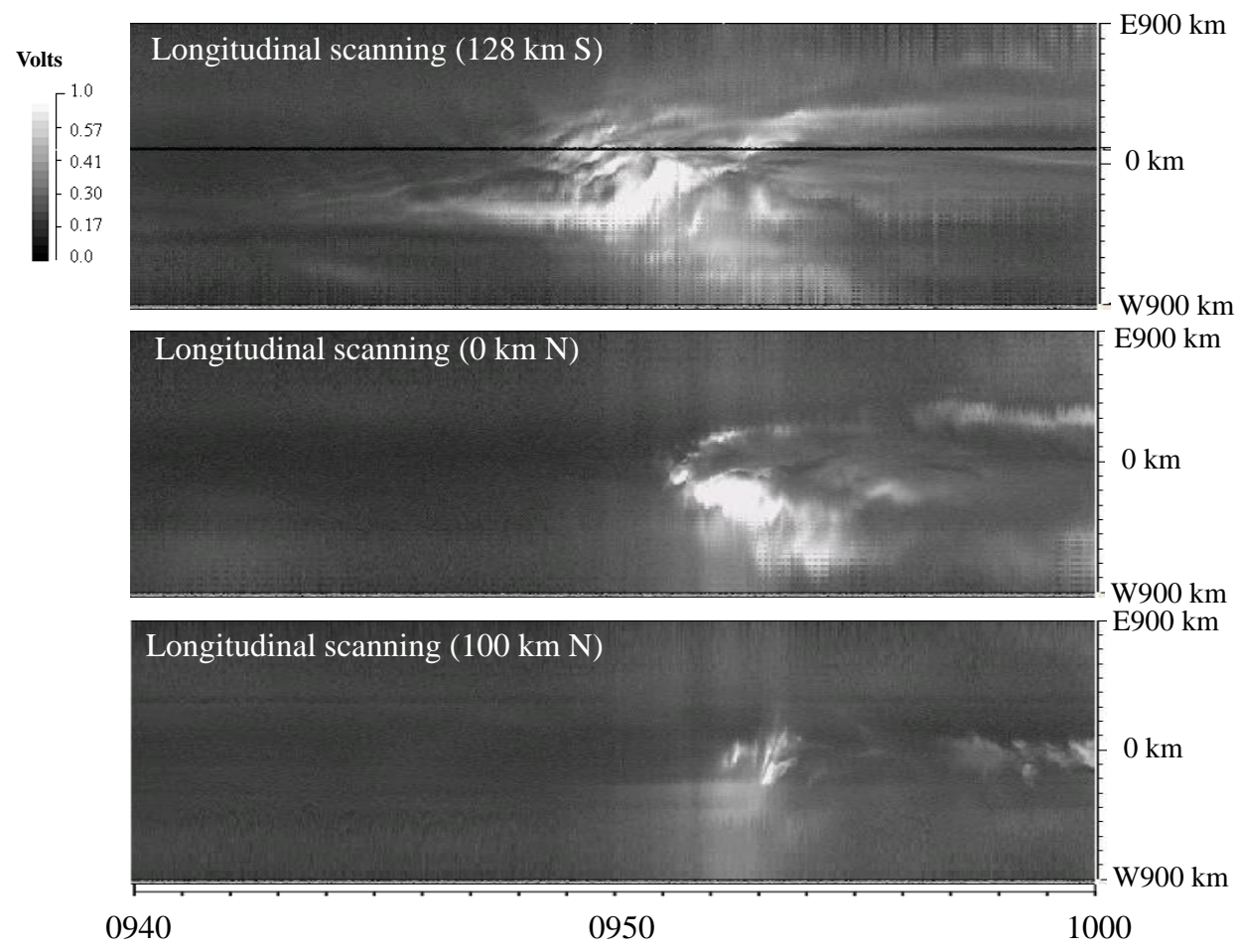

Figure 2. Longitudinal scanning of aurora along three different latitudes, $128 \mathrm{~km}$ south of DWS (128 km S), above DWS (0 km N), and $100 \mathrm{~km}$ north of DWS $(100 \mathrm{~km} \mathrm{~N})$ for the 20 min intervals from 09:40 UT to 10:00 UT. The longitudinal scanning is $1800 \mathrm{~km}$ from $900 \mathrm{~km}$ west of DWS and to $900 \mathrm{~km}$ east of DWS. The aurora gray level is plotted to the left. 


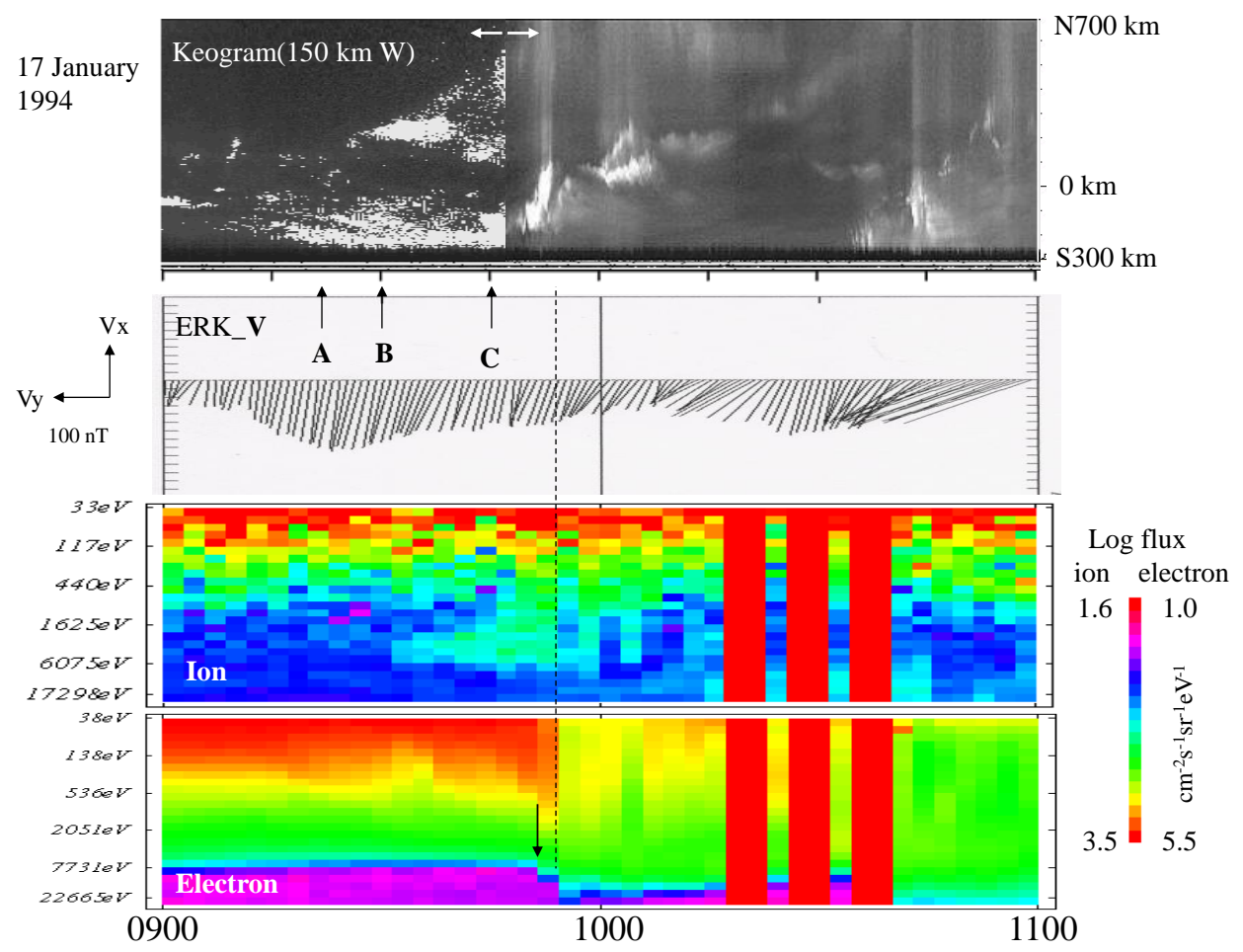

Figure 3. Same as Fig. 1, but showing a keogram $(150 \mathrm{~km} \mathrm{~W})$, the polar cap convections from ERK ( $88.7^{\circ}$ ILAT), the ion spectrogram from $33 \mathrm{eV}$ to $17.3 \mathrm{keV}$, and the electrons from $38 \mathrm{eV}$ to $22.7 \mathrm{keV}$. In the keogram, the auroras above 0.305 in the gray level ( 0 for black and 1 for white) were highlighted in bright white before 09:47 UT (marked by arrows), while the gray level in Fig. 1 is used after 09:47 UT. The polar cap convection is plotted in the sun-earth coordinates (see text). Vx denotes sunward, Vy denotes duskward. The time resolution is $1 \mathrm{~min}$. The vertical line in the velocity plots denotes the time mark at 10:00 UT. A spin-averaged flux in $\mathrm{cm}^{-2} \mathrm{~s}^{-1} \mathrm{sr}^{-1} \mathrm{eV}^{-1} \mathrm{obtained} \mathrm{in}$ one $\sim 10 \mathrm{~s}$ spin with repetition interval of $\sim 3 \mathrm{~min}$ is plotted. Logarithmic scales for the ion and the electron flux are to the right. Thick red vertical lines in the ion and the electron spectrogram denote data gaps. The arrow in the fourth panel denotes a step-like increase of the upper cutoff energies of the plasma-sheet electrons (see text).

\subsection{Plasma and auroral observations prior to the expansion onset}

Figure 3 shows, from top to bottom, the keogram $(150 \mathrm{~km} \mathrm{~W})$ of the auroras, the polar cap convections at ERK (88.7 $\left.{ }^{\circ} \mathrm{ILAT}\right)$, the ion flux spectrogram from $33 \mathrm{eV}$ to $17.3 \mathrm{keV}$, and the electron spectrogram from $38 \mathrm{eV}$ to $22.7 \mathrm{keV}$. The dotted lines also designate the first peak of the negative bay of DWS at 09:53 UT. Because the keogram contained pre-onset faint auroras and the following bright expansion auroras, the keogram prior to and after 09:47 UT, as marked by arrows, is shown using a different gray level. For the pre-onset auroras, signals above 0.305 in the gray level (0 for black and 1 for white) were highlighted in bright white, while for the expansion auroras the gray level in Fig. 1 was used. To plot the polar cap convections, the $H$ and $D$ components of ERK were first converted into sun-earth coordinates where the offset amplitudes of $48 \mathrm{~h}(-24$ to $+24 \mathrm{~h})$ means were subtracted. The field line vectors in sun-earth coordinates were rotated $90^{\circ}$ counterclockwise. The convection flows were assumed to be proportional to the rotated field line vectors. The anti-sunward convection thus deduced began in the polar cap at 09:13 UT, 40 min prior to the negative bay peak. Although the auroral activities began $90 \mathrm{~min}$ prior to the negative bay peak, in association with the increase in the electron flux below $1 \mathrm{keV}$ at the geosynchronous orbit (not shown), we focus on $T-40 \min$ to $T=0$, where $T=0 \mathrm{de}$ notes the time of the negative bay peak. For the $40 \mathrm{~min}$ intervals, ions in 1-6 keV were observed after 09:31 UT (see the third panel of Fig. 3). The arrow in the electron spectrogram in the fourth panel denotes a step-like increase of the upper cutoff energies of plasma-sheet electrons (Thomsen et al., 2002) from $6 \mathrm{keV}$ at 09:48:45 UT to $10 \mathrm{keV}$ at 09:51:37 UT, just prior to the peak of negative bay.

In the keogram $(150 \mathrm{~km} \mathrm{~W})$, equatorward propagating streaks repeated at about $120 \mathrm{~s}$ intervals were observed after 09:15 UT equatorward of $5100 \mathrm{~km}(100 \mathrm{~km}$ south of DWS). The propagating velocity was $0.13 \mathrm{~km} \mathrm{~s}^{-1}$ to the onset latitudes. At higher latitudes, an auroral band appeared after 09:30 UT between $\mathrm{N} 200 \mathrm{~km}$ to $\mathrm{N} 300 \mathrm{~km}$ (200 to $300 \mathrm{~km}$ north of DWS). Equatorward motions were not observed for this auroral band. 

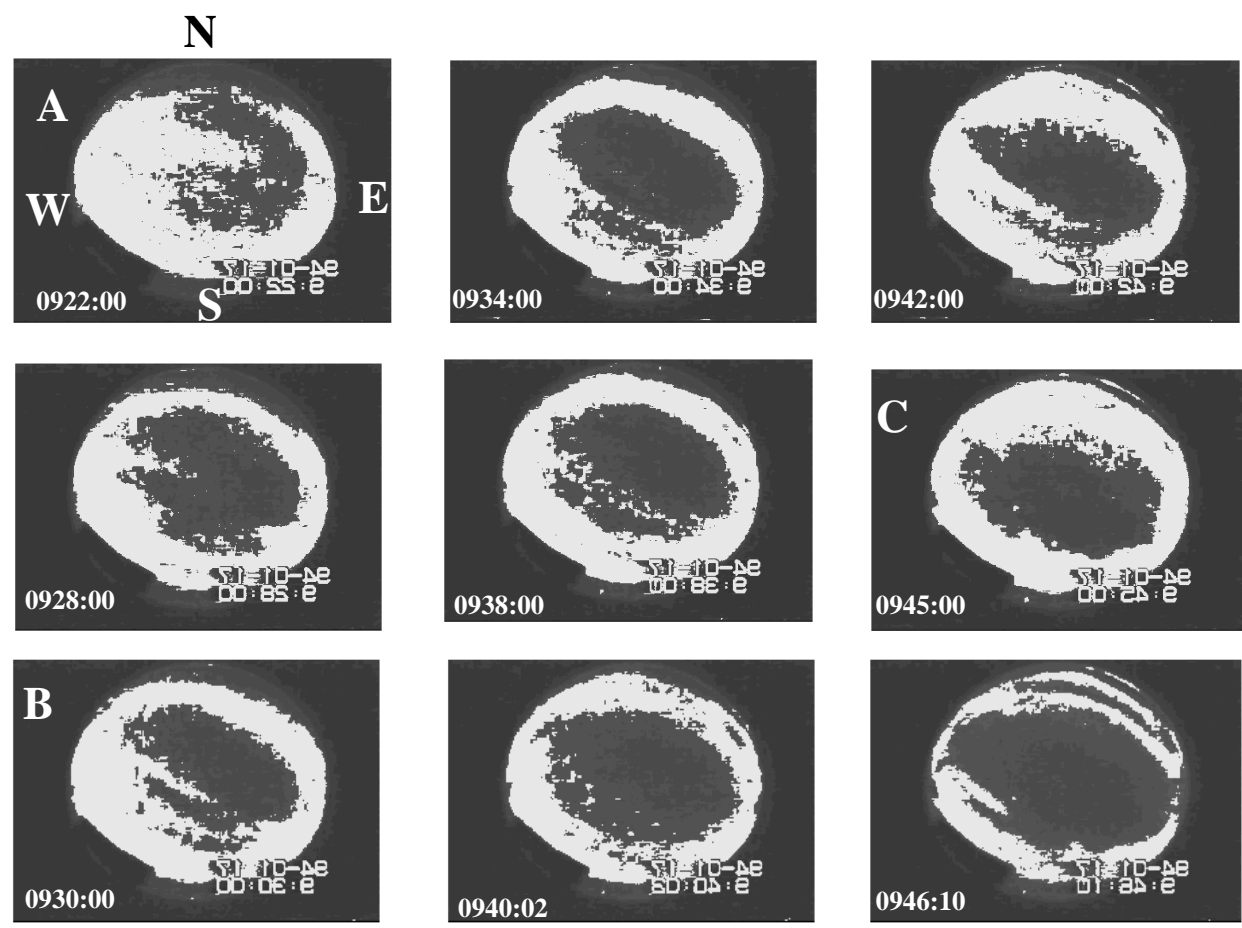

Figure 4. Nine all-sky images from 09:22:00 to 09:46:10 UT for 17 January 1994. The auroras above 0.322-0.355 in the gray level were highlighted. The images labeled A, B, and C were taken at A, B, and C in Fig. 3. East is to the right, west is to the left, north is up, and south is down. The sequence sampled at $\sim 10$ s from 09:20:00 to 09:47:50 UT is given in Animation_1 (see Supplement). Note that the gray level is reversed in the animation (darker colors for the brighter auroras) and were viewed from the ground, anti-parallel to the field lines. East is to the left and west to the right in the animation.
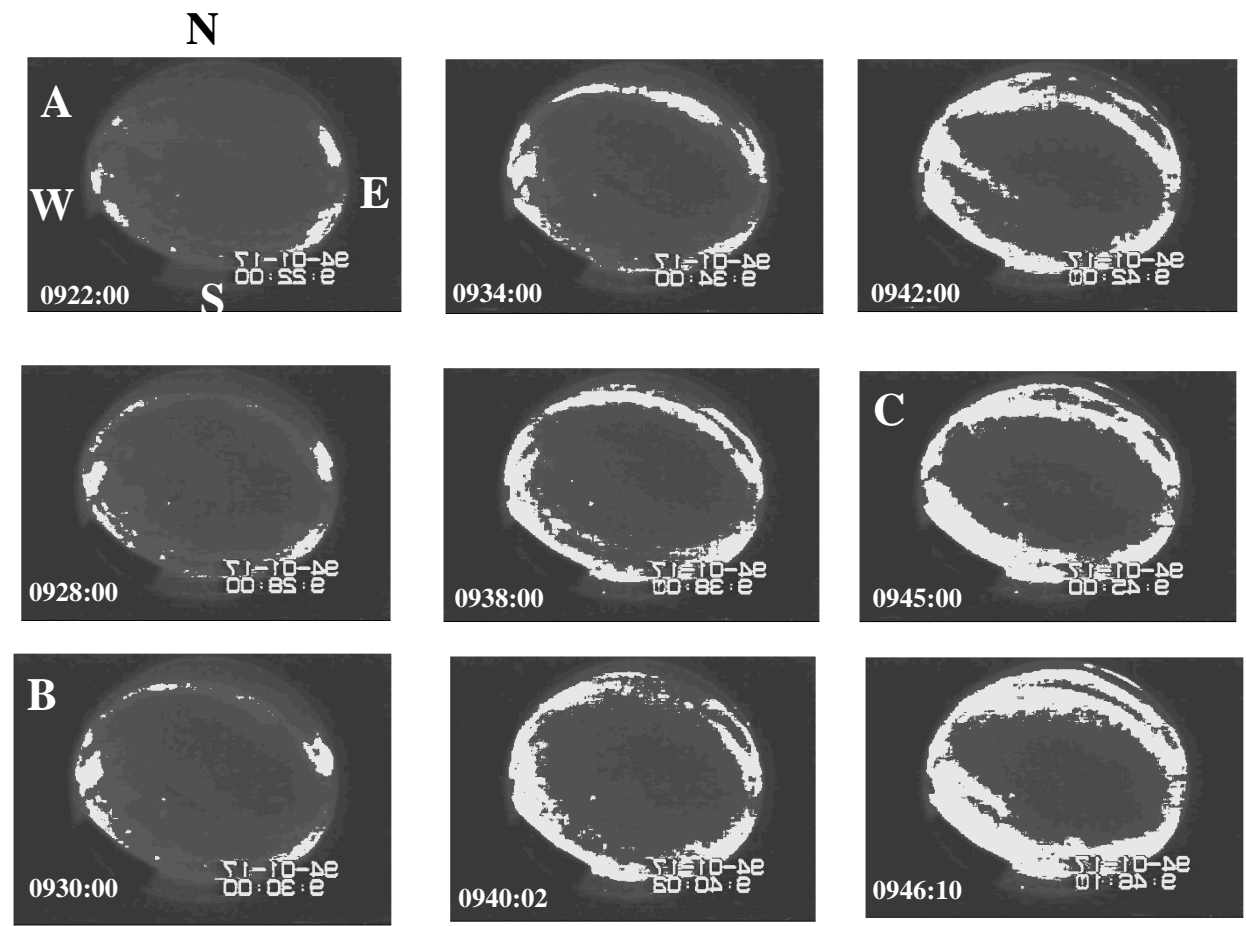

Figure 5. Same as Fig. 4 but the highlighted level is $0.350-0.370$ in the gray level, slightly higher than that in Fig. 4 (the wave-like structure in Fig. 4 disappeared because of the weaker radiance). 


\subsection{Chronological development of auroras in an all-sky image}

The chronological development of the auroras is displayed in Figs. 4, 5 and 7 via the all-sky images. Figures 4 and 5 demonstrate pre-onset faint auroras, where auroras above $0.322-0.370$ in the gray level were highlighted. The aurora images taken at 09:22:00 UT (A), 09:30:00 UT (B), and 09:45:00 UT (C) in Fig. 3 are correspondingly marked A, $\mathrm{B}$, and $\mathrm{C}$ in Figs. 4 and 5. The motion of the aurora in the animation (Animation_1 in the Supplement) better represents the pre-onset aurora than a still image. In the animation, the image gray level is reversed (darker color is for the brighter aurora), and is viewed anti-parallel to the field lines. East is to the left and west to the right in the animation. At about 09:22:31 UT (94-01-17 9:22:31 in Animation_1 in the Supplement), an auroral band appeared at the poleward boundary. Simultaneously, an auroral arc propagating southwestward appeared at the center. The arcs progressively developed and propagated southwestward until 9:42:30 (09:42:30 UT). Such propagations are also shown in the highlighted images in Fig. 4. The propagating auroral arcs were related to the three streaks propagating toward the onset latitudes in the keogram shown in Fig. 3. In order to show in detail the southwestward-propagating aurora arcs, the all-sky image taken at 09:29:22 UT is presented in Fig. 6. Two arcs were recorded in the image, one at the zenith and the other equatorward. They represent the wave front propagating southwestward at an angle of about 10 $20^{\circ}$ clockwise from the geomagnetic east-west directions. The westward velocity of the front was $\sim 1.5 \mathrm{~km} \mathrm{~s}^{-1}$. The equatorward velocities were $0.1-0.2 \mathrm{~km} \mathrm{~s}^{-1}$. The equatorward propagating streaks in the keogram can be regarded as equatorward-propagating high- $m$ waves $(T \sim 120 \mathrm{~s}, m \sim 76)$. The high- $m$ waves observed in Animation_1 (see Supplement) until 9:42:30 (09:42:30 UT) again appeared near the onset latitudes at 9:45:00. The auroral band at higher latitudes $(\mathrm{N} 200-\mathrm{N} 300 \mathrm{~km})$ showed westward propagations in Animation_1 (see Supplement) for the interval from 9:26:10 to $9: 38: 50$ at velocities of $\sim 0.3 \mathrm{~km} \mathrm{~s}^{-1}$. The westward velocity may correspond to the magnetic drift of the $7.7 \mathrm{keV}$ ions along $L=6.6$. It is likely that the auroral band at higher latitudes is associated with the proton auroras excited by the 1-6 keV ions simultaneously observed by the L9 satellites. The auroral surge was observed in Animation_1 (see Supplement) after 9:38:50 along the latitudes poleward of the auroral band. The poleward surge is demonstrated in the highlighted images in Fig. 5, showing the multiple bands at the poleward boundary.

At the onset latitudes, the activities first appeared in the western sector at 9:43:10 in Animation_1 (see Supplement) with the eastward propagations, which were followed by bead-like rippling (see the image taken at 09:49:42 UT in Fig. 7 and Animation_2 in the Supplement). The rotating motions in the bead-like rippling grew into multiple shear layers

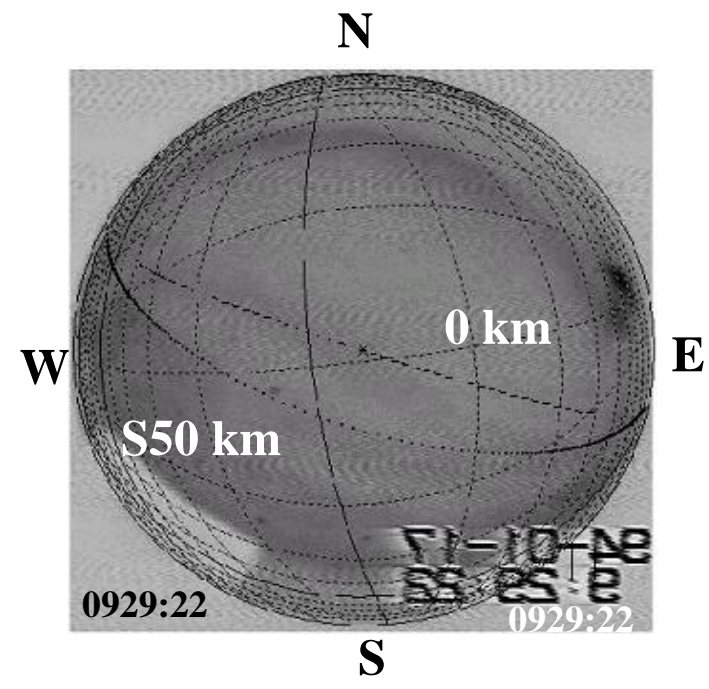

Figure 6. All-sky image taken at 09:29:22 UT overlaid by the lines of geographic longitudes and latitudes, and the geomagnetic eastwest lines passing the zenith of DWS $(0 \mathrm{~km})$ and $50 \mathrm{~km}$ south of DWS $(\mathrm{S} 50 \mathrm{~km})$ by dotted lines. The brighter part of the auroras is shown in darker colors. The arc rotated clockwise with respect to the geomagnetic east-west direction.

by 09:51:16 UT (Fig. 7), which displayed a clockwise rotation when viewed along the field lines from above the ionosphere (9:48:28-9:50:50 in Animation_2 in the Supplement). The breakup ends by the scattering of the shear layers, as observed in the image taken at 09:53:48 UT (Fig. 7). Later, a new surge emerged from the eastern sector, wandering along the lower latitudes.

\subsection{Auroral activations at the equatorward latitudes}

Figure 8 shows, from top to bottom, the auroral longitudescanning plot along the onset latitudes, the $H, D$, and $Z$ components of DWS for the interval 09:40 to 10:00 UT. Here, $H$, $D$, and $Z$ point toward magnetic north, magnetic east, and downward, respectively. A wave-like structure drifting westward was observed again for the intervals 09:43-09:49 UT and in the range E500-W200 km. The westward phase velocity tended to increase to about $5.6 \mathrm{~km} \mathrm{~s}^{-1}$. The high- $m$ waves lasted until the onset of the bead-like rippling. Pulsating auroras (the vertical streaks repeating at $\sim 10 \mathrm{~s}$ ) were activated further westward. The vertical arrows in the second panel mark the onsets of the bead-like rippling (09:48:20 UT), the multiple shear layers (09:52:29 UT), and the end of the poleward expansion (09:53:45 UT). The first bead-like rippling started its eastward propagation from $E 150 \mathrm{~km}$ at 09:48:20 UT. The onset longitudes of the subsequent beadlike rippling moved progressively westward. The propagating velocities of the bead-like rippling were $\sim 3 \mathrm{~km} \mathrm{~s}^{-1}$. An eastward drift on the same order of magnitude can also be 

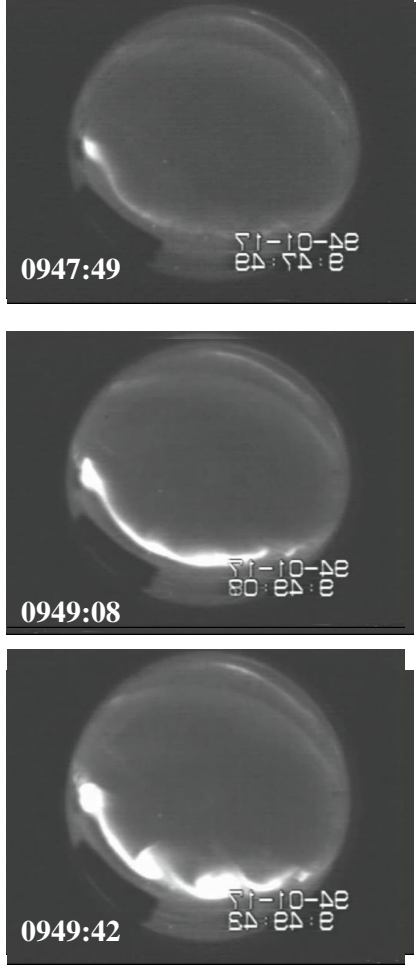
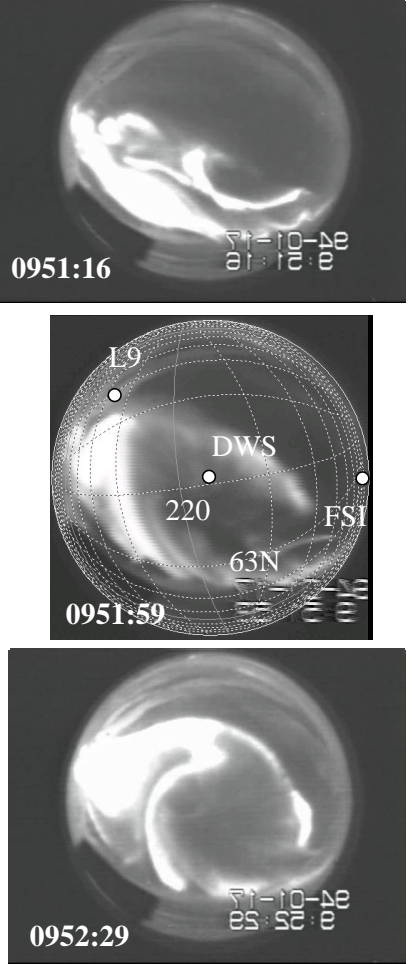
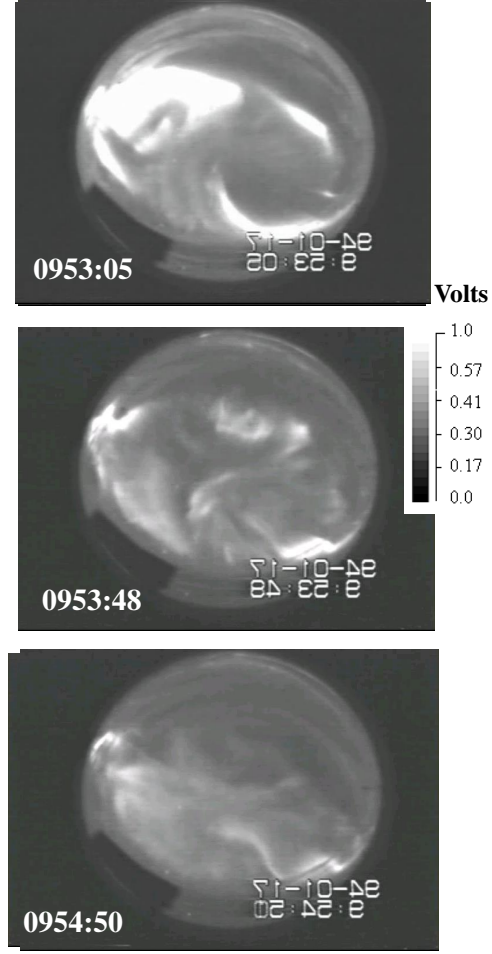

Figure 7. Nine all-sky images from 09:47:49 to 09:54:50 UT for 17 January 1994. The brighter part of the auroras is in white. Geographic latitudes and longitudes are overlaid on the center image (09:51:59) using grids. Latitudes are plotted every one degree, while the longitudes are for every two degrees. The satellite footprints calculated by the IGRF, the optical station DWS, and the ground magnetometer station FSI are overlaid by white circles. East is to the right, west is to the left, north is up, and south is down. The geomagnetic coordinates are rotated $19.6^{\circ}$ clockwise at DWS with respect to the geographic coordinates. The sequence sampled at $\sim 2 \mathrm{~s}$ from 09:48:00 to 09:55:01 UT is given in Animation_2 (see Supplement). Note that images in the animation were viewed anti-parallel to the field lines. East is to the left and west to the right in the animation.

observed for the interval 09:52:30-09:54:45 UT during the activation of the multiple shear layers.

\subsection{ULF activities on the ground}

The magnetic field data in the second through the fourth panels in Fig. 8 show that the first occurrence of the amplitude decrease of $H$ and the increase of $Z$ correlates to the onset of the bead-like rippling. The decrease in the amplitude of $D$ began slightly earlier. High- $m$ waves in the aurora occurring prior to the bead-like rippling were not observed by the ground magnetometer, most likely because of their short wavelength (high wave number). A sharp decrease in the $D$ component occurred at 09:51:17 UT, which correlated to the beginning of the poleward motion of the equatorward boundary of the aurora. The decrease of $H$ and the increase of $Z$ continued until 09:52:10 UT when the $Z$ component peaked. $Z$ decreased quickly to the pre-onset level by 09:52:35 UT. After a short interval, the $Z$ component decreased further below the pre-onset level and returned back to the pre-onset level (the level before 09:48 UT) by 09:53:52 UT. The $Z$ amplitude kept increasing until 09:54:50 UT. There occurred a step-like decrease of the $H$ component marked by arrows 1 to 2 and 3 to 4 . The first decrease accompanied the positive peak of $Z$ (I to II), whereas the second decrease accompanied the negative peak of $Z$ (III to IV). The zigzag-path of the $D$ component is denoted by arrows a to e. From the FFT power spectral analyses of the $H$ component in Fig. 9, the common frequencies in the $10-30 \mathrm{mHz}$ range are sheared from high latitudes (DWS, $\left.65.7^{\circ} \mathrm{N}\right)$, mid- $\left(\mathrm{PFO}, 39.5^{\circ} \mathrm{N}\right)$ to low latitudes (GUA, $4.5^{\circ} \mathrm{N}$ ), and from 19.7 MLT (GUA) to 1.7 MLT (PFO) in local time. The waveform plot for the $10 \mathrm{~min}$ intervals from 09:50 to 10:00 UT in Fig. 10 shows that the $H$ of the high-latitude station (DWS) was out of phase with respect to the $H$ of GUA. In lower latitudes, the eastern station (PFO) delayed the $H$ of the western station (GUA) by about $40 \mathrm{~s}$. Figure 11 demonstrates the waveform of the $H \mathrm{com}$ ponent in the auroral zone from the western station (KOT, 22.0 MLT) to the eastern station (FMC, 1.8 MLT) through DWS (23.4 MLT) and FSI (0.8 MLT) for the same intervals in Fig. 10. The main peak at DWS (09:53:20 UT) is marked by a solid line and moving east, while for the first peak, marked by a dotted line (09:52:10 UT), the eastern station (DWS) leads the western station (KOT). For the main peak, there appeared 

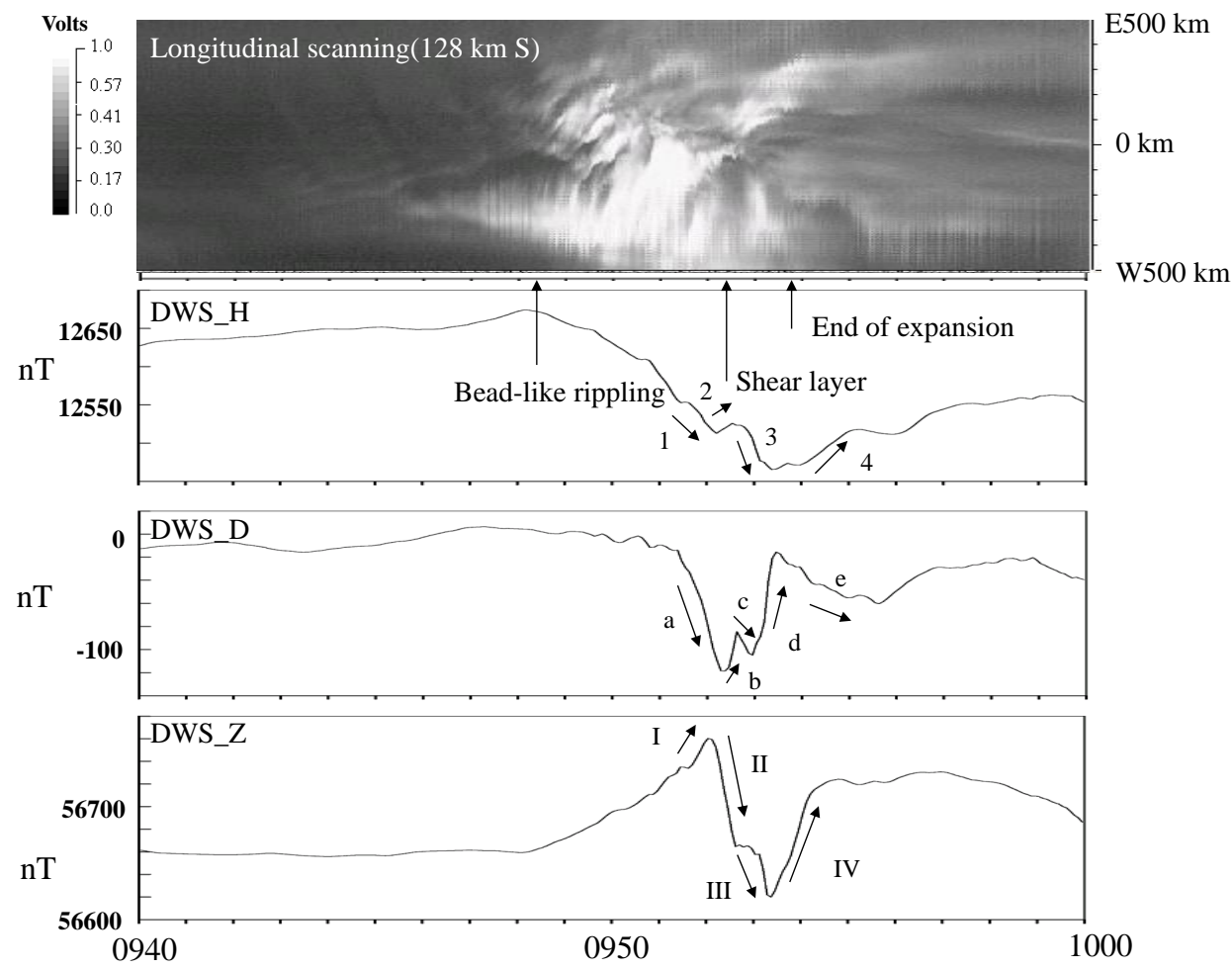

Figure 8. From top to bottom: 20 min plots of longitudinal scanning along the equatorward arc ( $128 \mathrm{~km}$ south of DWS), the $H$ component of the DWS magnetogram, the $D$ component for DWS, and the $Z$ component for DWS. The onset of the bead-like rippling, the onset of the multiple shear layers, and the end of the poleward expansion in the auroral display are shown by vertical arrows. The arrows labeled 1 to 4 for $H$, a to e for $D$, and I to IV for $Z$ in the second, third, and fourth panels, respectively, denote characteristic changes of the field line vectors associated with the passage of the ionospheric loop current (see text). The aurora gray level is plotted to the left.

a delay of $25 \mathrm{~s}$ between DWS and FSI. Such a delay in the main peak was also observed in the band-passed data in 10$30 \mathrm{mHz}$. The amplitudes of the pulse in the band-passed data were 20, 35, 2, and 2nT for KOT, DWS, FSI, and FMC, respectively. The time delays between longitudinally separated stations in the auroral zone (Fig. 11) and in lower latitudes (Fig. 10) may suggest an overall propagation of the magnetic pulse toward the east with sub-structures in the auroral zone. The auroral activities moving westward were found at the onset latitudes until the onset of the shear layer at about 09:52 UT (Fig. 8). It is likely that the initial negative pulse (09:52:10 UT, Fig. 11) is associated with this westward auroral motion. The main negative pulse (09:53:20 UT), however, propagated eastward.

The polarization ellipses in the $D-H$ plane for the intervals of 09:50-10:00 UT are plotted in Fig. 12, viewed along the field lines. The characteristic field changes marked by the arrows in Fig. 8 caused a polarization ellipse between gray circles. The polarization ellipse was rotated clockwise (CW) in the $D-H$ plane by the change in the field line vectors from 1 to 2 for the $H$ component and from a to $\mathrm{b}$ for the $D$ component. Subsequently, the polarization reversed to counterclockwise (CCW) by the field line changes 3 to 4 in $H$ and through c, d and e in $D$. The characteristic change of the field lines from a to e in $D$ is marked by arrows beside the polarization ellipses. The ground polarizations in Fig. 12 may be described by the divergence-free equivalent current systems in the ionosphere (Fukushima, 1976; Amm et al., 2002).

\subsection{Ionospheric currents associated with the auroral breakup}

In the upper-right inset in Fig. 13, the excitation of the ionospheric current vortex by the guided poloidal waves is proposed. In this model, the compressional input propagating eastward locally displaces the outer boundary in both the transverse and meridian planes and excites toroidal and poloidal modes, respectively (Allan et al., 1985). It is assumed that the inward compression generates the localized diamagnetic current $(\boldsymbol{J})$ flowing dusk-to-dawn in the equatorial plane during the passage of the compressional input at a velocity $(\boldsymbol{U})$. The currents invoke the third harmonic deformations of the field lines in the meridian plane (Saka et al., 2012b). The third harmonic in higher latitudes and fundamental harmonic in lower latitudes generate westward and eastward Hall currents in the ionosphere in higher and lower latitudes, respectively. Those currents close in the ionosphere through the meridional currents caused by the eastward 


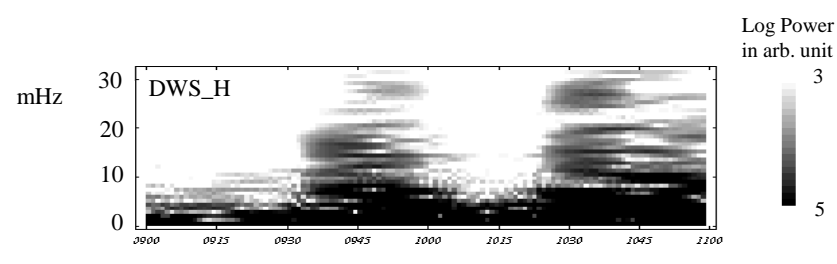

$\mathrm{mHz}$
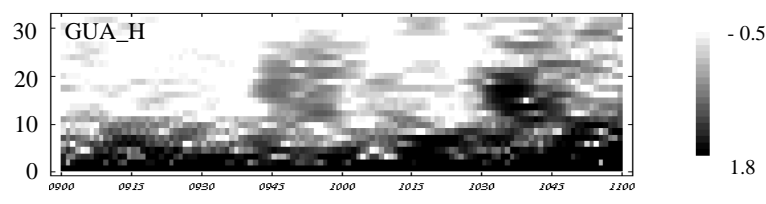

$\mathrm{mHz}$

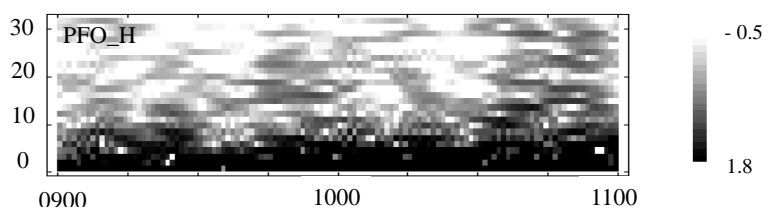

Figure 9. From top to bottom: the dynamic power spectra of $H$ for DWS, GUA, and PFO calculated by the FFT (fast Fourier transform) technique. The spectral power was calculated using 256 data points (12.8 $\mathrm{min}$ ) by sliding $1 \mathrm{~min}$.

(westward) deflections of the field lines in the leading edge and westward (eastward) deflections in the trailing edge of the compressional input propagating to the east (west). Ionospheric currents thus generated by the compressional input close counterclockwise when viewed from above.

The loop current closing counterclockwise at the ionospheric altitudes (not the equivalent current) is illustrated in the lower left of Fig. 13 by solid and dotted circles. The arrows overlaid on the loop current (solid circle) represent equivalent current vectors calculated from the ground effects of the loop current. Because of the symmetry of the current loop, only the poleward half is drawn. The upward fieldaligned current is placed at the center of the loop. The current loop (dotted circle) first grew in the western sector of DWS in association with the onset of the bead-like rippling, which correlated to a peak in the $H$ component and an initial rise in the $Z$ component. Then, a new current loop (dashed circle) from the east propagated past the DWS sector in association with the westward propagation of the first dip in the $H$ component (the positive peak of $Z$ ). In association with the eastward propagation of the second dip of $H$ (the negative peak of $Z$ ), the current loop (solid loop) approached from the west and propagated past the DWS sector. Propagation of the loop correlated to the onset of the poleward expansion (09:51:10 UT). From the $Z$ component profile, DWS can be located outside the loop during the westward propagation of the first current loop, whereas DWS crossed the loop during the eastward propagation of the second current loop. Prior to 09:51:10 UT, the characteristic changes in the field line vector exhibit linear polarization on the horizontal plane and a monotonic increase in the $Z$ component. We interpret this interval in terms of the growing current intensities of the current loop.

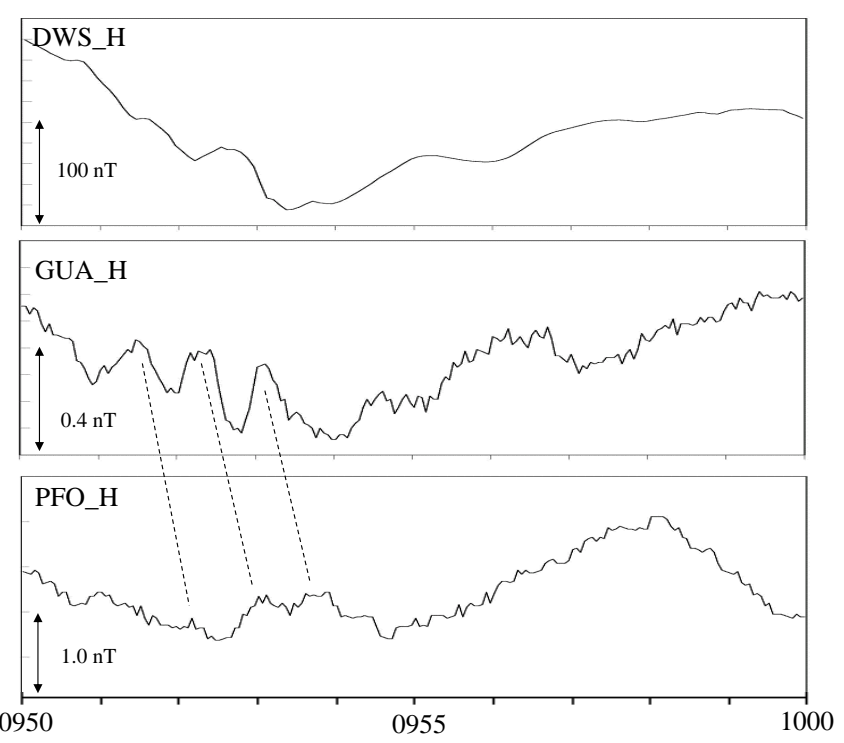

Figure 10. From top to bottom: plot of the waveform of $H$ for DWS, GUA, and PFO for the intervals from 09:50 UT to 10:00 UT. A possible delay of the waveform of PFO with respect to GUA is marked by a dotted line.

We simulated the ground perturbation fields by applying the Biot-Savart law to the single current loop. Figure 14 shows a change of field vector $(H, D$, and $Z)$ of the ground station associated with the east-west propagating ionospheric current loop closing counterclockwise. For the calculations, the radius of the current loop was set to $500 \mathrm{~km}$ with the height of the ionosphere at $100 \mathrm{~km}$. The ground image currents are neglected. To match the observations, the top panel (a) is for the dashed loop of the currents in Fig. 13, whereas the second panel (b) is for the solid loop of the currents propagating eastward. The top panel shows that the ground station (DWS), approaching from the west of the loop, observed a negative $H$, which peaked when DWS crossed the center meridian of the current loop. $D$ changed the polarity from positive to negative as the ground station moved to the eastern half of the current loop. $Z$ shows a positive peak when the station crossed the center meridian of the current loop. Panel (a) compared to panel (b) shows that $D$ reversed polarity in association with the change in the propagation direction of the current loop from westward to eastward, whereas $H$ was not affected by the propagation direction. As demonstrated in panel (b), $Z$ changed the amplitudes from positive to negative (or negative to positive) when the ground station crossed the edge of the loop. The polarization ellipse was determined by the propagation direction: $\mathrm{CW}$ for westward propagation and CCW for eastward propagation. $Z$ amplitudes were determined by the location of the ground station at the inside or the outside position of the loop. The observed field vector changes, 1 to 4 for $H$, a to e for $D$, and I to IV for $Z$, were simulated reasonably well by the propagating current loop closing counterclockwise. 


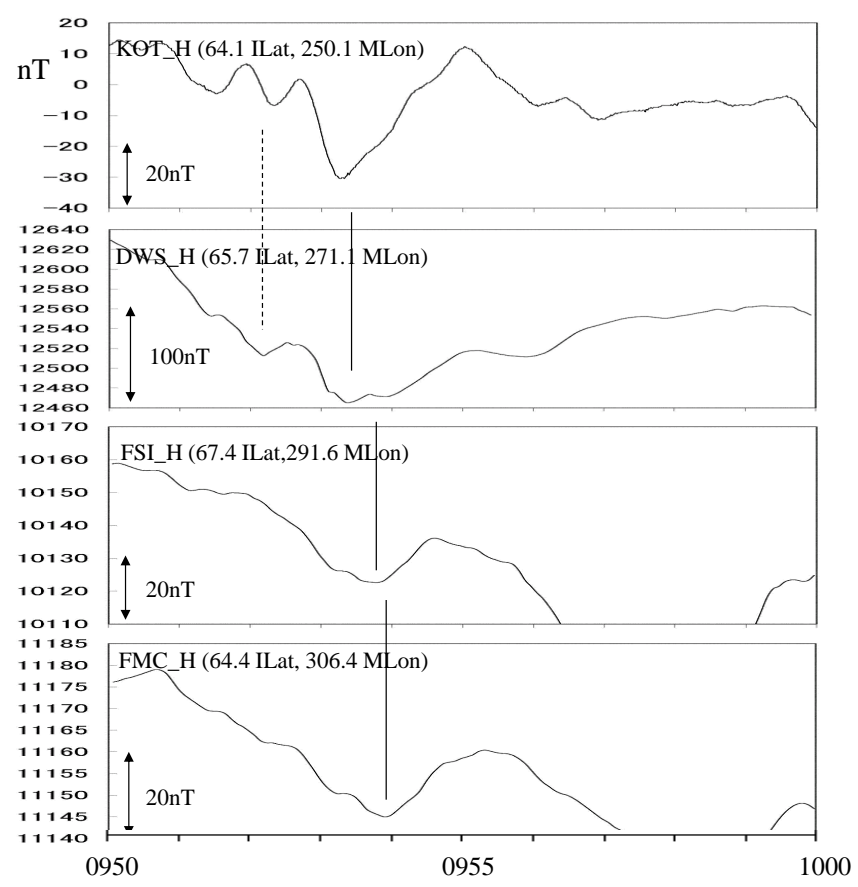

Figure 11. Same as Fig. 10 but for auroral zone stations of KOT, DWS, FSI, and FMC. Dotted and solid lines mark the peaks of the negative amplitudes of DWS at 09:52:10 and 09:53:20 UT, respectively. Peak of the negative pulse at FSI and FMC was at 09:53:45 and 09:53:50 UT, respectively. Time resolution of KOT was $1 \mathrm{~s}$, while resolutions were $5 \mathrm{~s}$ for DWS, FSI, and FMC.

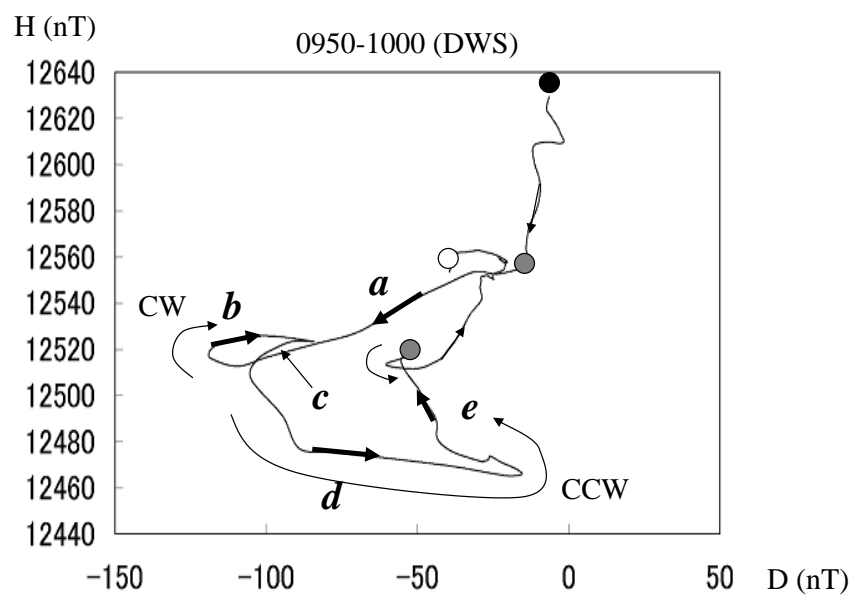

Figure 12. Hodogram of the polarization ellipses on the $D-H$ plane for the interval 09:50-10:00 UT. The plot starts from the black circle and ends at the white circle. The polarization ellipse between the gray circles was caused by characteristic changes of the field vectors, 1 to 4 in $H$, and a to e in $D$ in Fig. 8. The arrows mark the field line changes associated with a to e in $D$.

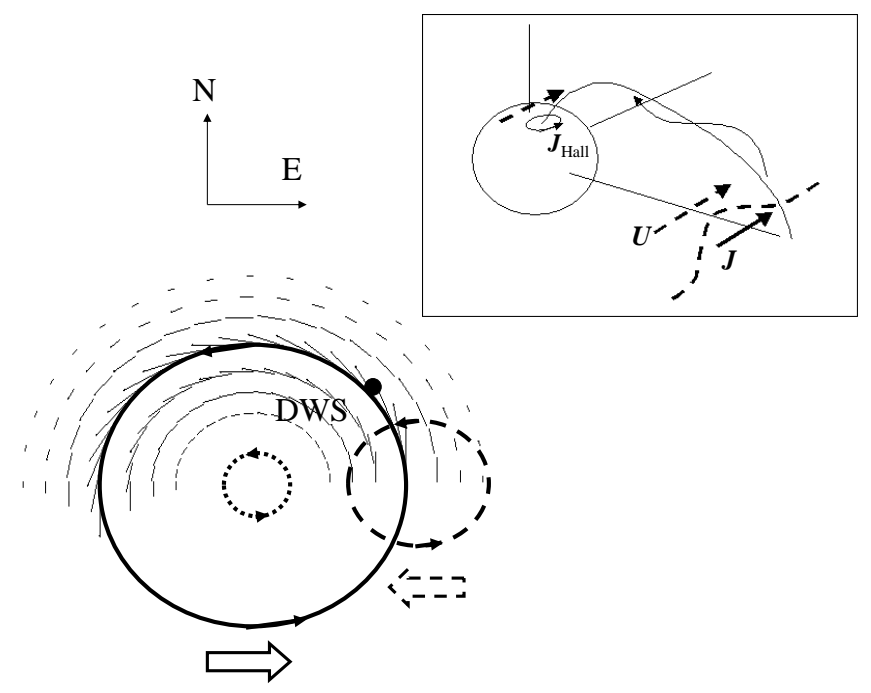

Figure 13. Schematic illustration of the ionospheric loop currents closing counterclockwise. Dotted circles illustrate a loop current growing in intensity. The current loop propagating westward is shown by a dashed circle. The solid circle represents the current loop propagating eastward. The dot indicates the ground magnetometer station (DWS). The equivalent ionospheric current vectors associated with the loop current shown by the solid line are overlaid. Only the vectors in the poleward half are presented. In the upper-right corner, generation of the ionospheric loop current is illustrated by the field line deformations associated with the compressional input propagating eastward along the outer boundary at the velocity $(\boldsymbol{U})$ (see text). Displaced surface of the compressional input is shown by the dotted curve. The compressional input accompanied the diamagnetic currents $(\boldsymbol{J})$ flowing eastward. The ionospheric loop current propagated eastward, following the eastward propagation of the compressional input.

\section{Discussion}

Following the increase of the electron flux $<1 \mathrm{keV}$ at geosynchronous altitudes observed by the L9 satellite, the enhancement of anti-sunward convection in the polar cap occurred $40 \mathrm{~min}$ prior to the onset of the negative pulse at DWS. A step-like increase in the upper cutoff energies of plasmasheet electrons occurred just before the negative pulse. The increase in the cutoff energies indicates an increasing convection electric field, which moved the open-close boundary of electron trajectories closer to the Earth (Thomsen et al., 2002). The surge of the earthward convection of the plasmasheet electrons correlated to the bead-like rippling at the onset latitudes. It is supposed that the plasma sheet approached the poleward boundary of the all-sky field of view and a part of the plasmas was transported earthward by the convection, which may have started before the onset of the convection surge. Auroral bands at higher latitudes may represent the earthward boundary of the plasma sheet. The high- $m$ waves propagated southwestward at velocities of $0.13 \mathrm{~km} \mathrm{~s}^{-1}$ to the south and $1.5 \mathrm{~km} \mathrm{~s}^{-1}$ to the west. The high- $m$ waves may be 


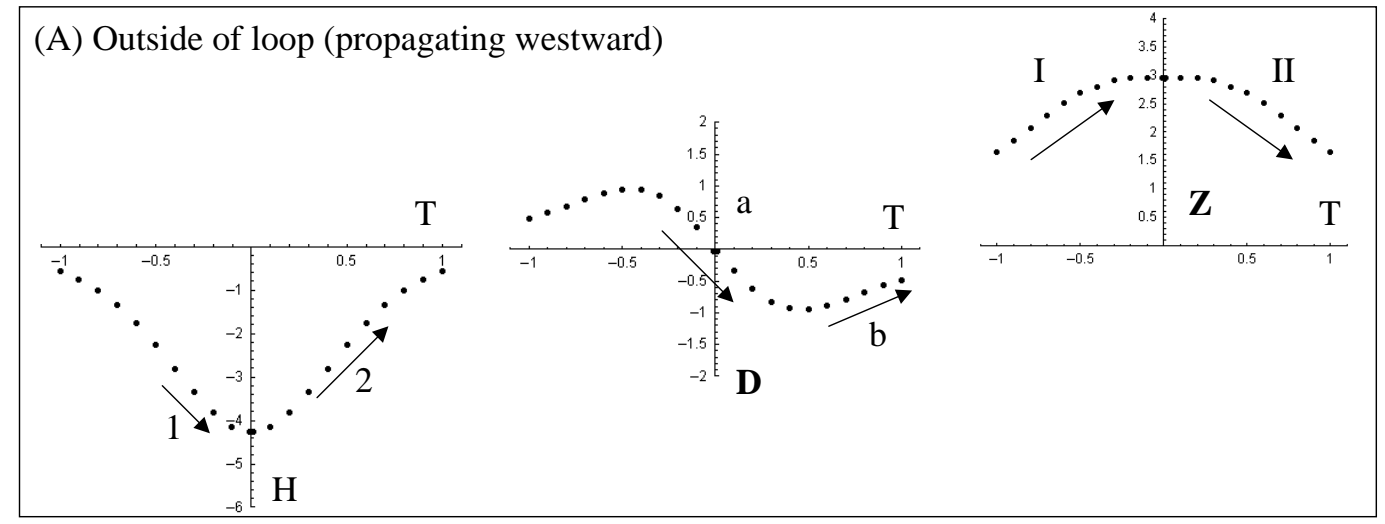

(B) Inside of loop (propagating eastward)

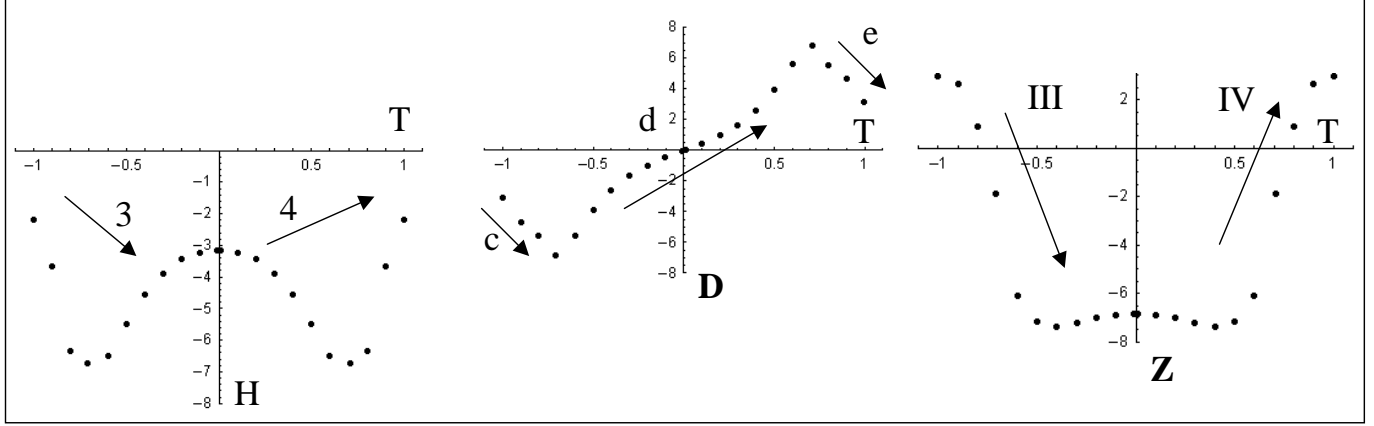

Figure 14. Calculated magnetic fields of $H, D$, and $Z$ components on the ground produced by the loop current propagating at ionospheric altitudes $(100 \mathrm{~km})$. The amplitudes are relative. The imaging currents of the ground are not included. The radius of the ionospheric current is assumed to be $500 \mathrm{~km}$. In (A), the ground station is located at $600 \mathrm{~km}$ north of the center of the loop; (B) represents the ground station located $350 \mathrm{~km}$ north of the center of the loop. The loop current approached from the east for $(\mathbf{A})$ and from the west for $(\mathbf{B})(T=-1)$, passed overhead $(T=0)$, and moved to the west or to the east of the current loop $(T=1)$. Arrows in the plots $(1$ to 4 for $H$, a to e for $D$, and I to IV for $Z$ ) correspond to the field line changes labeled in Fig. 8.

driven by internal energies (plasma-sheet plasmas) through drift-bounce resonance (Fenrich and Samson, 1997; Mann, 1998) or by drift resonance (Mager et al., 2009). In the magnetogram, both the $H$ and the $D$ components kept increasing during the intervals when the high- $m$ waves were observed. Then, a decrease in the $H$ and the $D$ components and an increase in the $Z$ component began in association with the onset of the bead-like rippling. The switch of the amplitude change from increasing to decreasing may represent the onset of the local activations of the ionospheric vortical currents.

The ionospheric loop current may have peak intensity at the sector of the maximum plasma pressures introduced in the conjugate magnetosphere by the energetic ion injection, which may correspond to the breakup sector on the ground. The eastward propagation of the main pulse at velocities of $0.8 \mathrm{deg} \mathrm{s}^{-1}$ (or $36 \mathrm{~km} \mathrm{~s}^{-1}$ ) (DWS to FSI in Fig. 11) and $30 \mathrm{~s}$ for the transit time of the loop (c to d of the $D$ component in Fig. 8) gives the diameter of the current loop on the order of $1080 \mathrm{~km}$. The loop size is consistent with the size of the aurora. With the propagation directions associated with this event, the loop current closing counterclockwise explained the ground observations. This result supports the idea that the propagating compressional input in the magnetosphere excited the ionospheric vortical currents. The vortical current may accompany the converging electric field in the ionosphere as a primary electric field. The secondary electric field generated by the non-uniform distributions of the conductivities in the ionosphere may modify the primary electric field (Glassmeier, 1984). A dense ground network may reveal the ionospheric current system in greater detail (Pashin et al., 1982; Glassmeier et al., 1988; Amm et al., 2002).

\section{Summary}

An isolated and localized auroral breakup event was studied using all-sky images, ground magnetometer data from optical station, and plasma data at the conjugate equatorial plane. The results obtained are as follows:

1. Wave-like structures were observed in the faint aurora during the pre-onset intervals beginning $30 \mathrm{~min}$ prior to the auroral breakup. The wave-like structures were characterized by the high- $m$ waves $(m \sim 76, T \sim 120 \mathrm{~s})$ propagating equatorward to the onset latitudes. 
2. The poleward expansion of the aurora accompanied the ground magnetic pulse. The magnetic pulse represents a propagating ionospheric loop current closing counterclockwise. The loop repeated at the Pi2 periodicity simultaneously observed in the mid- and low latitudes.

We conclude that the plasma-sheet plasmas transported from the tail generated the high- $m$ waves by the drift-bounce resonance or by the drift resonance, and thereby developed the current vortices for the auroral breakup.

\section{The Supplement related to this article is available online at doi:10.5194/angeo-32-1011-2014-supplement.}

Acknowledgements. The IGRF model calculations were performed through WDC for Aurora in NIPR. We acknowledge I. R. Mann, D. K. Milling and the rest of the CARISMA team for the DWS, FSI, and FMC data. CARISMA is operated by the University of Alberta, funded by the Canadian Space Agency. We also thank the CANMOS for the YKC, the 210 Magnetic Data for the KOT, the Equatorial Magnetometer Network for the GUA and PFO, and the WDC for Geomagnetism in Kyoto University.

Topical Editor L. Blomberg thanks T. Kornilova and one anonymous referee for their help in evaluating this paper.

\section{References}

Allan, W., White, S. P., and Poulter, E. M.: Magnetospheric coupling of hydromagnetic waves - initial results, Geophys. Res. Lett., 12, 287-290, 1985.

Allan, W., Menk, F. W., Fraser, B. J., Li, Y., and White, S. P.: Are low-latitude Pi2 pulsations cavity/waveguide modes?, Geophys. Res. Lett., 23, 765-768, 1996.

Amm, O., Engebretson, M. J., Hughes, T., Newitt, L., Viljanen, A., and Watermann J.: A traveling convection vortex event study: Instantaneous ionospheric equivalent current, estimation of fieldaligned currents, and the role of induced currents, J. Geophys. Res., 107, 1334, doi:10.1029/2002JA009472, 2002.

Baumjohann, W. and Glassmeier K.: The transient response mechanism and Pi2 pulsations at substorm onset-Review and outlook, Planet. Space Sci., 32, 1361-1370, 1984.

Birn, J., Thomsen, M. F., Borovsky, J. B., Reeves, G. D., McComas, D. J., and Belian, R. D.: Characteristic plasma properties during dispersionless substorm injections at geosynchronous orbit, J. Geophys. Res., 102, 2309-2324, 1997.

Bradshaw, E. G. and Lester, M.: SABRE observations of Pi2 pulsations: case studies, Ann. Geophys., 15, 40-53, doi:10.1007/s00585-997-0040-3, 1997.

Fenrich, F. R. and Samson, J. C.: Growth and decay of field line resonances, J. Geophys. Res., 102, 20031-20039, 1997.

Fukushima, N.: Generalized theorem for no ground magnetic effect of vertical currents connected with Pedersen currents in the uniform-conductivity ionosphere, Rept. Ionos. Space Res. Japan, 30, 35-45, 1976.

Glassmeier, K. H.: On the influence of ionospheres with nonuniform conductivity distribution on hydromagnetic waves, J. Geophys., 54, 125-137, 1984.
Glassmeier, K. H., Baumjohann, W., Korth, A., and Gough, P.: High-latitude Pi2 pulsations, ELF intensity, and particle flux variations: A case study, Ann. Geophys., 6, 287-296, 1988, http://www.ann-geophys.net/6/287/1988/.

Keiling, A. and Takahashi, K.: Review of Pi2 models, Space Sci. Rev., 161, 63-148, 2011.

Kim, K.-H, Lee, D.-H., Takahashi, K., Russel, C. T., Moon, Y.-J., and Yumoto, K.: Pi2 pulsations observed from the Polar satellite outside the plasmapause, Geophys. Res. Lett., 32, L18102, doi:10.1029/2005GL023872, 2005.

Lee, D.-H.: On the generation mechanism of $\mathrm{Pi} 2$ pulsations in the magnetosphere, Geophys. Res. Lett., 25, 583-586, 1998.

Lee, D.-H. and Lysak, R.: Impulsive excitation of ULF waves in the three-dimensional dipole model: the initial results, J. Geophys. Res., 96, 3479-3486, 1991.

Lester, M., Hughes, W. J., and Singer, H. J.: Polarization patterns of Pi2 magnetic pulsations and the substorm current wedge, J. Geophys. Res., 88, 7958-7966, 1983.

Mager, P. N., Klimushkin, D. Yu., and Ivchenko, N.: On the equatorward phase propagation of high- $m$ ULF pulsations observed by radars, J. Atmos. Sol. Terr. Phys., 71, 1677-1680, 2009.

Mann, I. R.: An MHD model for driven high $\mathrm{m}$ field line resonances, Geophys. Res. Lett., 25, 1515-1518, 1998.

Nishimura, Y., Lyons, L. R., Kikuchi, T., Angelopoulos, V., Donovan, E., Mende, S., Chi, P. J., and Nagatsuma, T.: Formation of substorm Pi2: A coherent response to auroral streamers and currents, J. Geophys. Res., 117, A09218, doi:10.1029/2012JA017889, 2012.

Pashin, A. B., Baumjohann, W., Raspopov, O. M., Yahnin, A. G., Opgenoorth, H. J., and Glassmeier, K. H.: Pi2 magnetic pulsations, auroral break-ups, and the substorm current wedge: a case study, J. Geophys., 51, 223-233, 1982.

Quinn, J. M. and Southwood, D. J.: Observations of parallel ion energization in the equatorial region, J. Geophys. Res., 87, 1053610549, 1982.

Radoski, H. R.: Highly asymmetric MHD resonances: the guided poloidal mode, J. Geophys. Res., 72, 4026-4027, 1967.

Radoski, H. R.: A theory of latitude dependent geomagnetic micropulsations: the asymptotic fields, J. Geophys. Res., 79, 595603, 1974.

Rae, I. J., Murphy, K. R., Miles, D. M., Watt, C. E. J., and Mann, I. R.: Comment on "Formation of substorm Pi2:A coherent response to auroral streamers and currents" by Y. Nishimura et al., J. Geophys. Res., 118, 3488-3496, doi:10.1002/jgra.50336, 2013.

Rostoker, G. and Samson, J. C.: Polarization characteristics of Pi2 pulsations and implications for their source mechanisms: location of source regions with respect to the auroral electrojet, Planet. Space Sci., 29, 225-247, 1981.

Saka, O., Watanabe, O., Okada, K., and Baker, D. N.: A slow mode wave as a possible source of Pi 2 and associated particle precipitation: a case study, Ann. Geophys., 17, 674-681, doi:10.1007/s00585-999-0674-4, 1999.

Saka, O., Hayashi, K., and Koga, D.: Periodic aurora surge propagating eastward/westward at poleward boundary of aurora zone during the first $10 \mathrm{~min}$ intervals of Pi2 onset, J. Atmos. Sol. Terr. Phys., 80, 285-295, doi:10.1016/j.jastp.2012.02.010, 2012a.

Saka, O., Hayashi, K., and Koga, D.: Excitation of the third harmonic mode in meridian planes for $\mathrm{Pi} 2$ in the auroral zone, 
J. Geophys. Res., 117, A12215, doi:10.1029/2012JA018003, 2012b.

Sakurai, T. and McPherron, R. L.: Satellite observations of Pi2 activity at synchronous orbit, J. Geophys. Res., 88, 7015-7027, 1983.

Samson, J. R.: Pi2 pulsations: High latitude results, Planet. Space Sci., 30, 1239-1247, 1982.

Samson, J. C. and Harrold, B. G.: Characteristic time constants and velocities of high-latitude Pi2's, J. Geophys. Res., 90, 1217312181, 1985.

Shiokawa, K., Yumoto, K., and Olson, J. V.: Multiple auroral brightenings and associated Pi2 pulsations, Geophys. Res. Lett., 29, 1537, doi:10.1029/2001GL014583, 2002.

Solovyev, S. I., Baishev, D. G., Barkova, E. S., Molochushkin, N. E., and Yumoto, K.: Pi2 magnetic pulsations as response on spatiotemporal oscillations of auroral arc current system, Geophys. Res. Lett., 27, 1839-1842, 2000.

Southwood, D. J. and Hughes, W. J.: Concerning the structure of Pi2 pulsations, J. Geophys. Res., 90, 386-392, 1985.
Sutcliffe, P. R. and Nielsen, E.: STARE observations of Pi2 pulsations, Geophys. Res. Lett., 17, 603-606, 1990.

Sutcliffe, P. R. and Nielsen, E.: The ionospheric signature of Pi2 pulsations observed by STARE, J. Geophys. Res., 97, 1062110636, 1992.

Takahashi, K., Ohtani, S.-I., and Yumoto, K.: AMPTE CCE observations of $\mathrm{Pi} 2$ pulsations in the inner magnetosphere, Geophys. Res. Lett., 19, 1447-1450, 1992.

Teramoto, M., Nose, M., and Sutcliffe, P. R.: Statistical analysis of Pi2 pulsations inside and outside the plasmasphere observed by the polar orbiting DE-1 satellite, J. Geophys. Res., 113, A07203, doi:10.1029/2007JA012740, 2008.

Thomsen, M. F., Korth, H., and Elphic, R. C.: Upper cutoff energy of the electron plasma sheet as a measure of magnetospheric convection strength, J. Geophys. Res., 107, 1331, doi:10.1029/2001JA000148, 2002.

Uozumi, T., Yumoto, K., Kawano, H., Yoshikawa, A., Ohtani, S., Olson, J. V., Akasofu, S.-I., Solovyev, S. I., Vershinin, E. F., Liou, K., and Meng, C.-I.: Propagation characteristics of Pi2 magnetic pulsations observed at ground high latitudes, J. Geophys. Res., 109, A08203, doi:10.1029/2003JA009898, 2004. 